\title{
GALNT14 genotype is associated with perineural invasion, lymph node metastasis and overall survival in resected cholangiocarcinoma
}

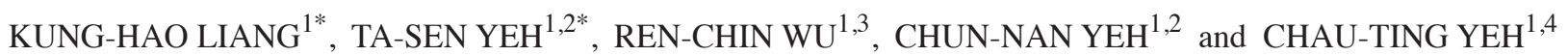 \\ ${ }^{1}$ Liver Research Center and ${ }^{2}$ Department of General Surgery, Chang Gung Memorial Hospital; \\ ${ }^{3}$ Department of Pathology, Chang Gung Memorial Hospital and Chang Gung University; \\ ${ }^{4}$ Molecular Medicine Research Center, Chang Gung University, Taoyuan 333, Taiwan, R.O.C.
}

Received January 25, 2016; Accepted January 31, 2017

DOI: $10.3892 / 01.2017 .5991$

\begin{abstract}
Cholangiocarcinoma is a rare, sporadic and aggressive type of cancer. The genetic basis of cholangiocarcinoma remains poorly understood. The present study investigated the prognostic role of the $\mathrm{N}$-acetylgalactosaminyltransferase 14 (GALNT14)-rs9679162 genotype, an effective therapeutic response predictor for hepatocellular carcinoma in patients with cholangiocarcinoma receiving surgical resection. A cohort of patients with intrahepatic or perihilar cholangiocarcinoma $(\mathrm{n}=112)$ were retrospectively recruited. Of these patients, 31.3, 49.1 and 19.6\% had GALNT14 'TT', 'TG' and 'GG' genotypes, respectively. The patient's genotype distributions did not deviate significantly from those of the ethnic reference cohorts, HapMap-Chinese Han Beijing and Chinese Han Metropolitan Denver. The genotype 'TT' was associated with unfavorable overall survival in univariate analysis $(\mathrm{P}=0.023)$. Furthermore, two tumor characteristics, perineural and vascular invasion, were independently associated with unfavorable overall survival $(\mathrm{P}=0.001$ and $\mathrm{P}=0.002$, respectively). The 'TT' genotypes were independently associated with two known predictors of unfavorable prognosis, perineural invasion $(\mathrm{P}=0.035)$ and lymph node metastasis $(\mathrm{P}=0.005)$ in a multivariate linear regression analysis. When compared with the two reference genotype cohorts, the 'TT' genotype was significantly higher in patients with perineural invasion ( $\mathrm{P}=0.049$, Beijing cohort; $\mathrm{P}=0.034$, Denver cohort). Similar enrichment of the 'TT' genotype was also revealed in patients with lymph node metastasis $(\mathrm{P}=0.046$, Beijing cohort; $\mathrm{P}=0.032$
\end{abstract}

Correspondence to: Dr Chau-Ting Yeh, Liver Research Center, Chang Gung Memorial Hospital, 5 Fu-Sing Street, Kuei-Shan Township, Taoyuan 333, Taiwan, R.O.C.

E-mail: chautingy@gmail.com

*Contributed equally

Key words: bile duct cancer, tumor etiology, single nucleotide polymorphism, glycosyltransferase
Denver cohort). In conclusion, the GALNT14-rs9679162 'TT' genotype was associated with perineural invasion and lymph node metastasis, as well as unfavorable overall survival in patients with resected cholangiocarcinoma.

\section{Introduction}

Cholangiocarcinoma is the second most common type of primary liver cancer worldwide, following hepatocellular carcinoma (HCC) (1). A higher number of mortalities are ascribed to cholangiocarcinoma, compared with HCC, in England and Wales since mid-1990 (1,2). Cholangiocarcinoma is rare in the majority of Western countries, and the rate of incidence ranges from $0.35 / 100,000$ in Canada to 3.36/100,000 in Italy (2). Conversely, the reported incidences are significantly higher in certain areas of Asia, including 5.7-85.0/100,000 in Thailand, $7.45-7.55 / 100,000$ in China, $7.10-8.75 / 100,000$ in Korea and $\sim 3.05-3.40 / 100,000$ in Japan (2). In Taiwan, a modest incidence of cholangiocarcinoma at 4.7/100,000 has been reported (2).

Cholangiocarcinoma emerges from the dysregulated proliferation of bile duct epithelial cells, known as cholangiocytes, and is notorious for its poor prognosis and response to chemotherapy (3). Clinically, cholangiocarcinoma is comprised of a group of tumors with markedly heterogeneous morphology, histology and clinical presentation (3). Cholangiocarcinoma may be classified as intrahepatic and extrahepatic types (1-3). The extrahepatic tumor is further classified into perihilar (Klatskin tumor) and distal forms (3). However, in certain cancer registries and epidemiological studies, perihilar cholangiocarcinoma has been considered as an intrahepatic tumor (4).

The etiology of cholangiocarcinoma remains largely unknown (4). Risk factors for cholangiocarcinoma include old age, primary sclerosing cholangitis, biliary tree stones and structural anomalies of bile ducts and liver flukes; however, combined, they account for $<30 \%$ of cholangiocarcinoma cases (1). Numerous molecular changes have been identified in cholangiocarcinoma, including the inactivation of tumor suppressor genes [tumor protein 53, anaphase-promoting complex, mothers against decapentaplegic homolog 4 (SMAD4) and cyclin dependent kinase inhibitor 2A], somatic 
mutations or the upregulation of oncogenes [e.g. Kirsten rat sarcoma (KRas), $c-M y c$ and human epidermal growth factor receptor $2(E R B B 2)$ ], and other chromosomal anomalies (2).

Surgical resection is applicable to $<40 \%$ of all intrahepatic cholangiocarcinoma cases $(1,5)$. Transcatheter arterial chemoembolization (TACE), radiofrequency ablation and combination chemotherapy (gemcitabine + cisplatin), have been used for the treatment of unresectable and recurrent cholangiocarcinoma $(1,2)$. A number of clinicopathological and genetic parameters have been identified as poor prognostic factors following surgical resection, including lymph node metastasis (1), positive resection margin (6), perineural invasion (7) and KRas mutations (8). No cancer staging systems and standard of care guidelines have been globally accepted $(9,10)$. However, four tumor characteristics, including vascular invasion, tumor number, lymph node metastasis and distant metastasis, are the major determinants in two staging systems developed independently in Japan and the United States to address the post-resection survival of intrahepatic cholangiocarcinoma $(9,10)$. Three staging systems are available for perihilar cholangiocarcinoma, including the American Joint Committee on Cancer Tumor-Node-Metastasis (TNM) system (11), the Bismuth-Corlette staging system (12) and the Blumgart modifications (13). All of these staging systems correlated poorly with post-resection survival in an earlier validation study (14).

Previously, through the use of the genome-wide association method followed by prospective validation, it was revealed that the germline genotypes of polypeptide $\mathrm{N}$-acetylgalactosaminyltransferase 14 (GALNT14) may serve as response predictors for chemotherapy in HCC (15). A leading single nucleotide polymorphism, rs9679162, was identified to be associated with chemotherapy response, time-to-tumor progression and overall survival in a previous study of patients with HCC at Barcelona Clinic Liver Cancer (BCLC) Stage C (16,17). The genotypes were also identified to correlate with the therapeutic response in TACE-treated patients with HCC at BCLC Stage B (18). It was revealed that the gene product of GALNT14 was an enzyme catalyzing O-glycosylation of numerous proteins, including the death receptors (DRs) 4 and 5 (19). O-glycosylation of DR 4/5 increased their sensitivity to extrinsic apoptotic signals (19). Furthermore, germline mutations in GALNT14 were associated with an increased risk of hereditary neuroblastoma (20) and GALNT14 was recently identified as an embryonic lethal gene based on studies in consanguineous families (21). Therefore, the association between the GALNT14 genotype and tumor behavior may not be restricted to HCC. The present study examined the association between the prognosis of patients with resected cholangiocarcinoma and the GALNT14 genotype.

\section{Materials and methods}

Patients. Under approval of the Institutional Review Board of Chang Gung Memorial Hospital (Taoyuan, Taiwan ROC), surgical tissue samples from 112 patients with cholangiocarcinoma, resected between January 1999-December 2008, were retrieved from the hospital's tissue bank, without any specific selection criteria. Written informed consent was obtained from all patients enrolled in the present study. Patients' clinical data were subsequently collected (see Table I), including age, sex, hepatitis B virus (HBV) surface antigen (HBsAg), anti-hepatitis C antibody (anti-HCV), cirrhosis, Eastern Co-operative Oncology Group performance status, biliary tree stones, cholangitis, tumor characteristics (location, invasion to vessels, perineural invasion, periductal invasion, lymph node metastasis, tumor number and size), histology, extrahepatobiliary invasion, resection margin and the extent of surgical resection. Pre-surgery biochemical data was collected, including on carcinoembryonic antigen (CEA), carbohydrate antigen 19-9 (CA-19-9), bilirubin, aspartate transaminase (AST) and alanine transaminase (ALT).

GALNT14 genotyping. GALNT14 genotyping was performed on thawed surgical tissue samples, which were freshly cryopreserved at $-70^{\circ} \mathrm{C}$ immediately following surgery. DNA was extracted from the tissues using QIAamp DNA Mini and Blood Mini kits (Qiagen GmbH, Hilden, Germany) following the manufacturers' protocol. Polymerase chain reactions were performed using a pair of primers (5'-TCACGAGGCCAAC ATTCTAG-3' and 5'-TTAGATTCTGCATGGCTCAC-3') to amplify the DNA fragment containing GALNT14-rs9679162 $\left(95^{\circ} \mathrm{C}, 1 \mathrm{~min} ; 55^{\circ} \mathrm{C}, 1 \mathrm{~min} ; 72^{\circ} \mathrm{C}, 1 \mathrm{~min} ; 30\right.$ cycles$)$, followed by direct sequencing using the conventional Sanger sequencing method (22). To ensure the accuracy of genotyping for each sample, polymerase chain reaction was performed two times and bidirectional sequencing was carried out.

Statistical analysis. Parametric data is presented as mean \pm standard deviation. Dichotomous data is presented as percentage. The genotype counts of GALNT14-rs9679162 in HapMap Chinese Han Beijing (CHB) and Metropolitan Denver (CHD) cohorts were retrieved from the public domain (http:// hapmap.ncbi.nlm.nih.gov/). These counts were compared with those obtained from the present study. Associations between the GALNT14 rs9679162 genotypes and clinical factors were analyzed using univariate and multivariate linear regressions. Genotype distributions were compared using the Cochran-Armitage Trend test or $\chi^{2}$ test. Loss of follow up was considered as censored data. Post-resection overall survival was analyzed using log-rank tests, Kaplan-Meier plots and the Cox proportional hazards model, where the censorship data occurred prior to the earliest events were dropped automatically by default of the SPSS Statistics 13.0 statistical software (SPSS Inc., Chicago, IL, USA). Statistical significance in the Cox proportional hazards model was evaluated using Wald tests. All tests were two-tailed. $\mathrm{P}<0.05$ was considered to indicate a statistically significant difference.

\section{Results}

Germline GALNT14 genotypes and tumor characteristics associated with overall survival in resected cholangiocarcinoma. Clinicopathological parameters of 112 patients with surgically resected cholangiocarcinoma are summarized in Table I. Major features of this cohort were as follows: HBsAg-negative (76.8\%), anti-HCV-negative $(86.6 \%)$, non-cirrhotic $(83 \%)$, non-HCC-cholangiocarcinoma-mixed histology $(87.5 \%)$ and intrahepatic $(77.7 \%)$. The frequency 
Table I. Clinical and tumor characteristics of the 112 patients included in the study.

\begin{tabular}{|c|c|}
\hline Parameters & Values \\
\hline Age, years, mean \pm SD & $60.2 \pm 10.7$ \\
\hline Sex, male $(\%)$ & $62(55.4)$ \\
\hline HBsAg, positive (\%) & $26(23.2)$ \\
\hline Anti-HCV, positive (\%) & $15(13.4)$ \\
\hline Cirrhosis, positive (\%) & $19(17.0)$ \\
\hline \multicolumn{2}{|l|}{ ECOG stage } \\
\hline 0 & $68(60.7)$ \\
\hline 1 & $44(39.3)$ \\
\hline Biliary tree stones, yes $(\%)$ & $24(21.4)$ \\
\hline Stone-unrelated cholangitis, yes (\%) & $46(41.0)$ \\
\hline \multicolumn{2}{|l|}{ Tumor characteristics } \\
\hline Perihilar, yes (\%) & $25(22.3)$ \\
\hline Invasion to vessel, yes (\%) & $30(26.8)$ \\
\hline Perineural invasion, yes $(\%)$ & $48(42.9)$ \\
\hline Periductal invasion, yes (\%) & $45(40.2)$ \\
\hline Lymph node involvement, yes (\%) & $33(29.5)$ \\
\hline \multicolumn{2}{|l|}{ Tumor number } \\
\hline 1 & $101(90.2)$ \\
\hline 2 & 6 \\
\hline 3 & 1 \\
\hline$>3$ & 4 \\
\hline Tumor size, $\mathrm{cm}$, mean $\pm \mathrm{SD}$ & $6.0 \pm 3.2$ \\
\hline \multicolumn{2}{|l|}{ Histology } \\
\hline Well differentiated, yes (\%) & $25(22.3)$ \\
\hline Mixed hepatocellular carcinoma, yes (\%) & $14(12.5)$ \\
\hline Extrahepatobiliary invasion, yes (\%) & $46(41.1)$ \\
\hline Resection margin involvement, yes (\%) & $44(39.3)$ \\
\hline $\begin{array}{l}\text { More than one segment of resection, } \\
\text { yes }(\%)\end{array}$ & $98(87.5)$ \\
\hline \multicolumn{2}{|l|}{ Biochemistry } \\
\hline $\mathrm{CEA}, \mathrm{ng} / \mathrm{ml}$, mean $\pm \mathrm{SD}$ & $41.0 \pm 104.4$ \\
\hline $\mathrm{CA}-19-9, \mathrm{U} / \mathrm{ml}$, mean $\pm \mathrm{SD}$ & $8,648.5 \pm 26,889.6$ \\
\hline Bilirubin, $\mathrm{mg} / \mathrm{dl}$, mean $\pm \mathrm{SD}$ & $1.8 \pm 3.1$ \\
\hline $\mathrm{AST}, \mathrm{U} / 1$, mean $\pm \mathrm{SD}$ & $56.3 \pm 69.5$ \\
\hline $\mathrm{ALT}, \mathrm{U} / \mathrm{l}$, mean $\pm \mathrm{SD}$ & $62.9 \pm 83.4$ \\
\hline \multicolumn{2}{|l|}{ GALNT14 genotype } \\
\hline $\mathrm{TT}(\%)$ & $35(31.3)$ \\
\hline TG $(\%)$ & $55(49.1)$ \\
\hline GG $(\%)$ & $22(19.6)$ \\
\hline
\end{tabular}

HBsAg, hepatitis B virus surface antigen; $\mathrm{HCV}$, hepatitis $\mathrm{C}$ virus; ECOG, Eastern Co-operative Oncology Group; CEA, carcinoembryonic antigen; CA-19-9, carbohydrate antigen 19-9; AST, aspartate aminotransferase; ALT, alanine aminotransferase; SD, standard deviation.

of the GALNT14-rs9679162 genotypes 'TT', 'TG' and 'GG' were $31.3 \%, 49.1 \%$ and $19.6 \%$, respectively (Fig. 1A), which did not deviate significantly from the ethnic reference genotype distribution of the HapMap Chinese Han Beijing (CHB)
A

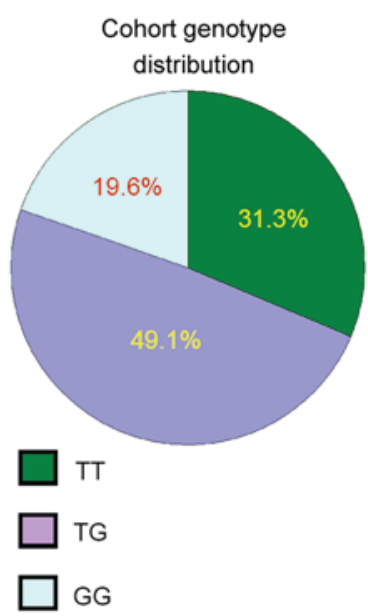

B

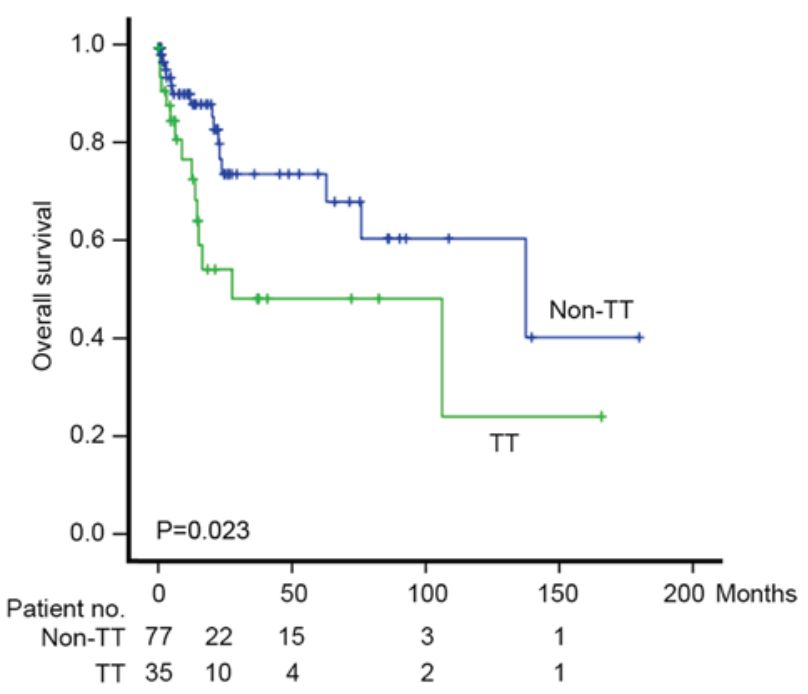

C

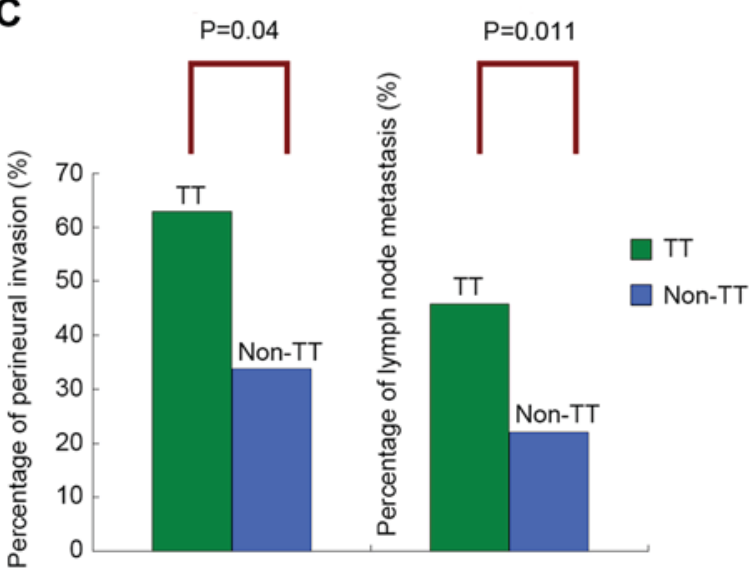

Figure 1. (A) GALNT14-rs9679162 genotype distribution in the study cohort. (B) Postoperative overall survival for cholangiocarcinoma stratified by patient genotype ('TT' vs. 'non-TT'; log-rank, $\mathrm{P}=0.023$ ). (C) Percentages of patients with perineural invasion and lymph node metastasis in the 'TT' and 'non-TT' subgroups. GALNT14, N-acetylgalactosaminyltransferase 14.

and Metropolitan Denver (CHD) cohorts (Cochran-Armitage Trend test, $\mathrm{P}=0.59$ and $\mathrm{P}=0.46$, respectively) (23).

Subsequently, clinicopathological parameters and GALNT14 genotypes were determined to be correlated with 


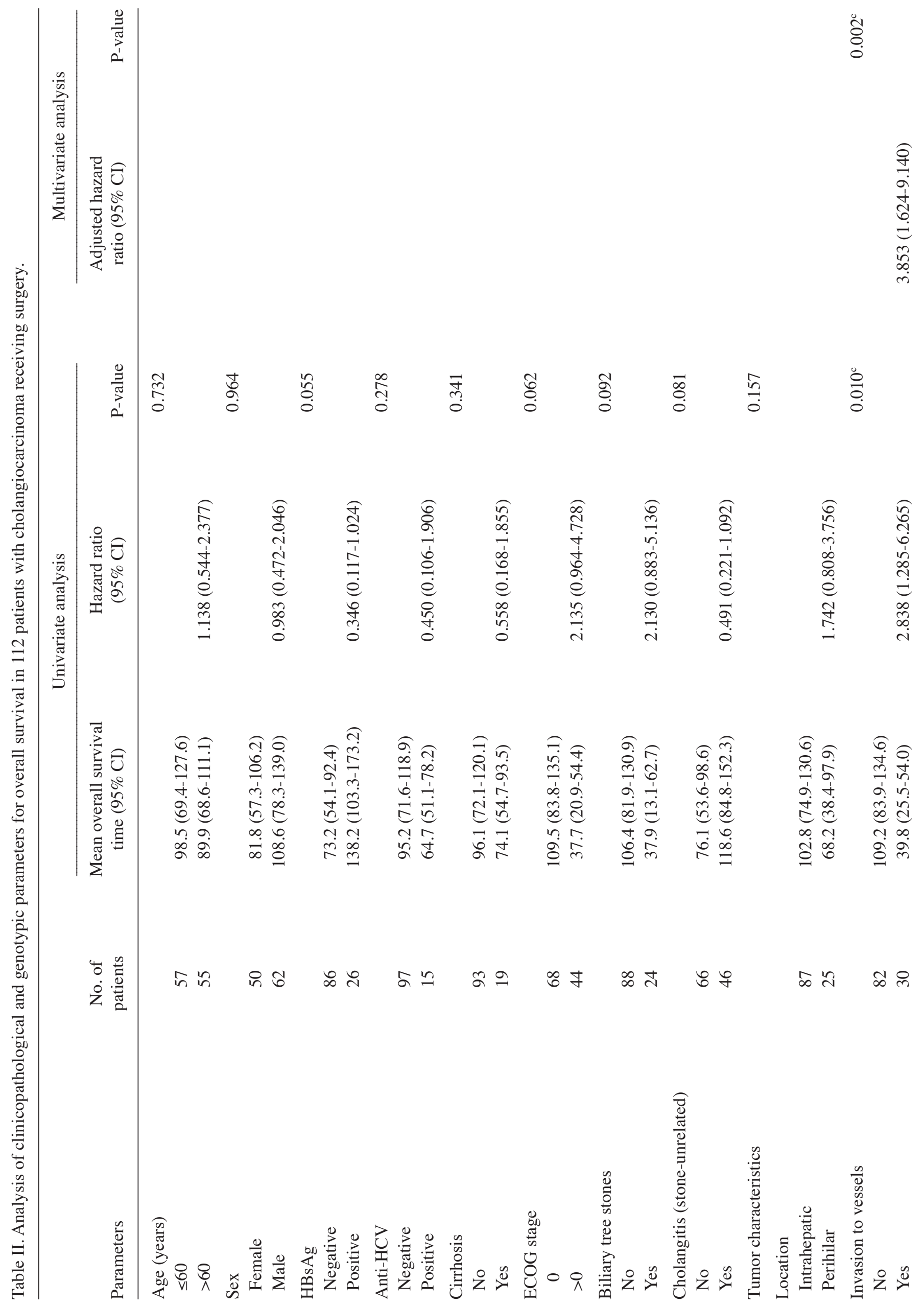




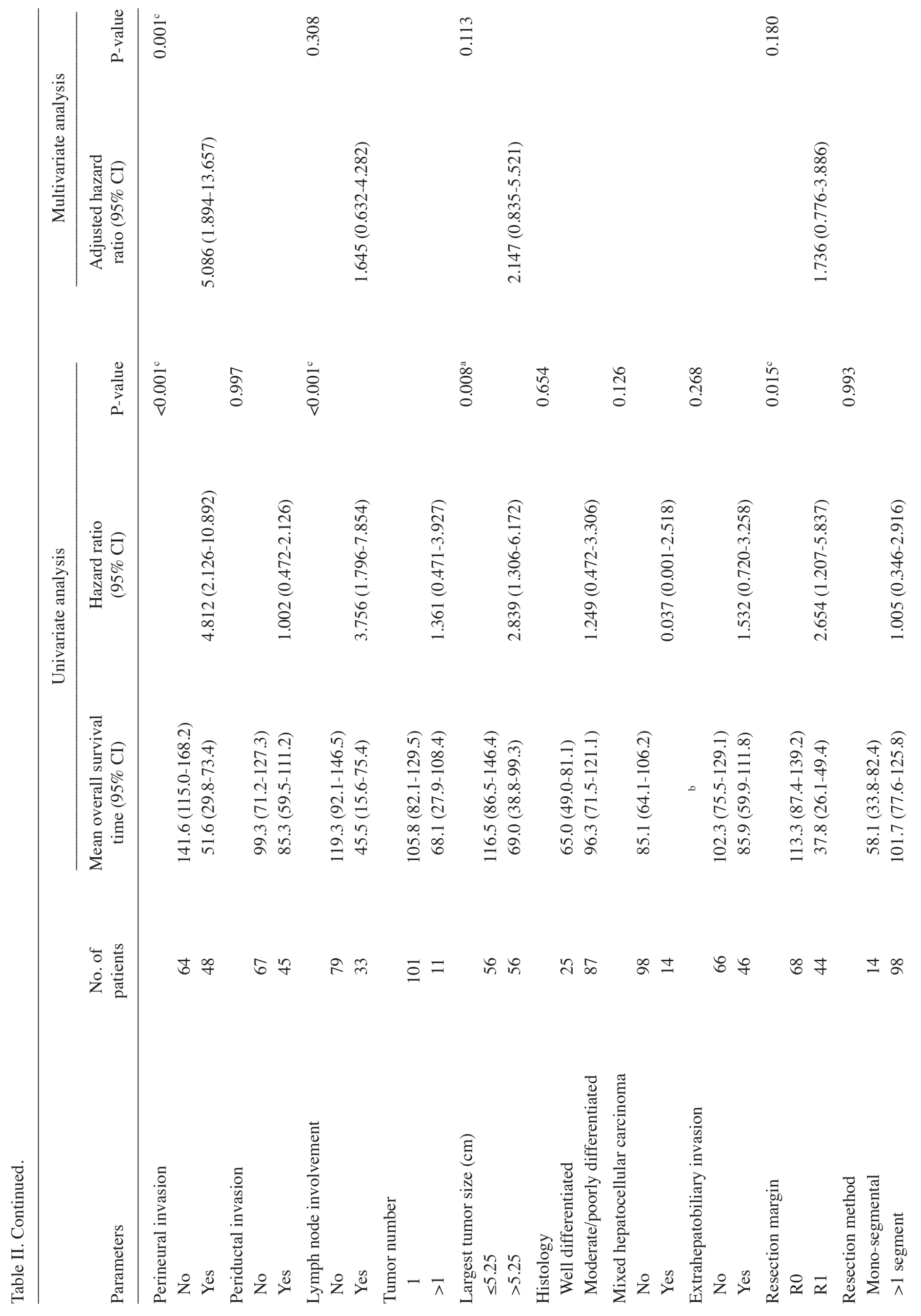




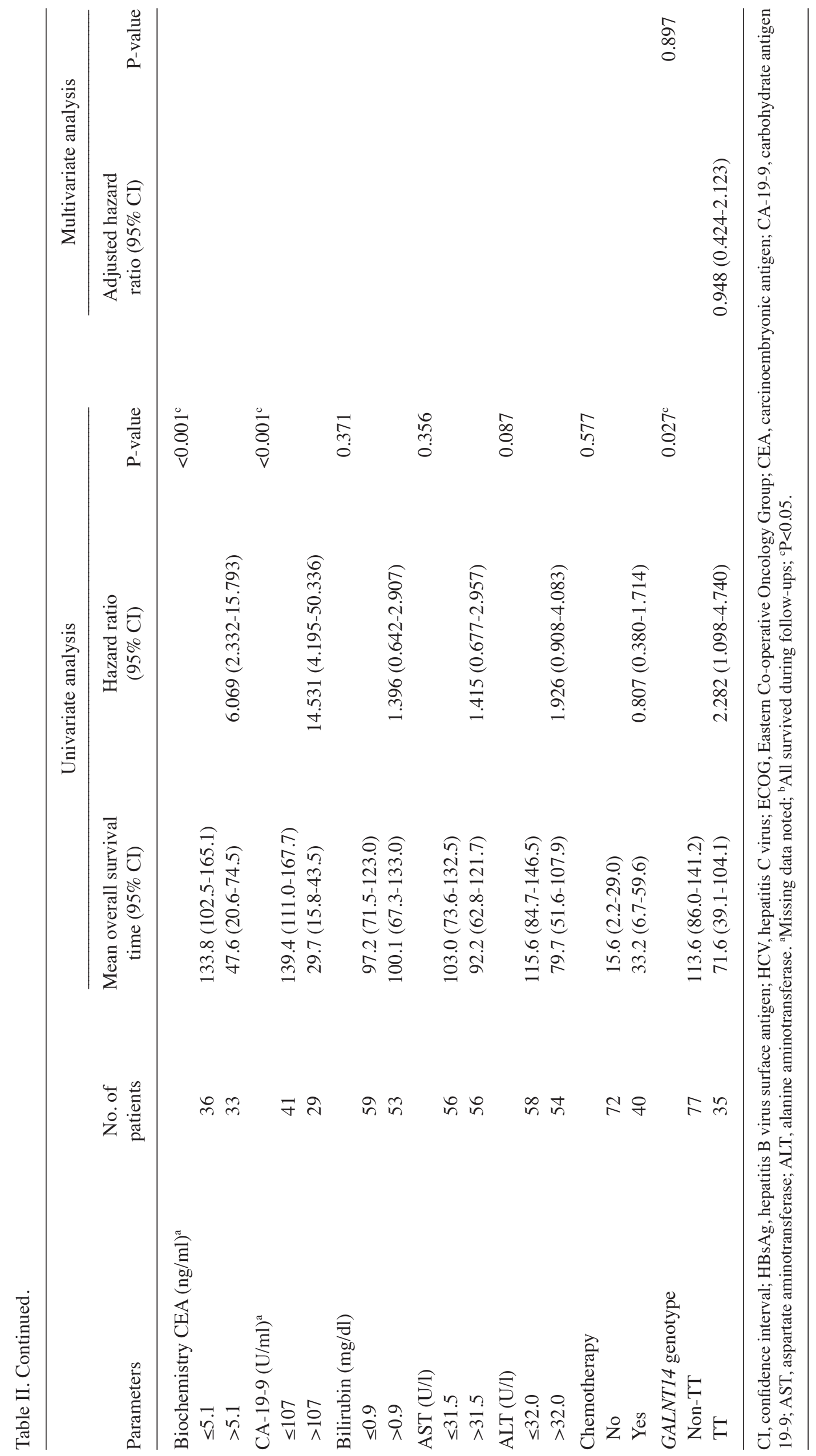


Table III. Linear regression analysis for correlation between clinicopathological factors and the GALNT14 'TT' genotype.

\begin{tabular}{|c|c|c|c|c|c|c|}
\hline \multirow[b]{2}{*}{ Parameters } & \multicolumn{3}{|c|}{ Univariate analysis } & \multicolumn{3}{|c|}{ Multivariate analysis } \\
\hline & $\beta$ & $95 \% \mathrm{CI}$ of $\beta$ & P-value & $\beta$ & $95 \% \mathrm{CI}$ of $\beta$ & P-value \\
\hline Sex, male & 0.059 & $-0.117-0.235$ & 0.510 & & & \\
\hline Age, years & -0.003 & $-0.011-0.005$ & 0.438 & & & \\
\hline HBsAg-positive & -0.006 & $-0.214-0.201$ & 0.952 & & & \\
\hline Anti-HCV-positive & -0.053 & $-0.310-0.204$ & 0.684 & & & \\
\hline ECOG status & -0.103 & $-0.281-0.075$ & 0.255 & & & \\
\hline Cirrhosis & 0.067 & $-0.166-0.300$ & 0.568 & & & \\
\hline Biliary tree stones & 0.080 & $-0.133-0.292$ & 0.461 & & & \\
\hline Cholangitis (stone-unrelated) & 0.068 & $-0.112-0.247$ & 0.456 & & & \\
\hline \multicolumn{7}{|l|}{ Tumor characteristics } \\
\hline Perihilar & 0.113 & $-0.097-0.322$ & 0.288 & & & \\
\hline Invasion to vessels & 0.120 & $-0.077-0.316$ & 0.231 & & & \\
\hline Perineural invasion & 0.255 & $0.085-0.425$ & $0.004^{\mathrm{a}}$ & 0.185 & $0.014-0.357$ & $0.035^{\mathrm{a}}$ \\
\hline Periductal invasion & -0.002 & $-0.181-0.176$ & 0.979 & & & \\
\hline Lymph node involvement & 0.060 & $0.027-0.093$ & $0.001^{\mathrm{a}}$ & 0.050 & $0.015-0.084$ & $0.005^{\mathrm{a}}$ \\
\hline Tumor number $>1$ & 0.158 & $-0.135-0.450$ & 0.289 & & & \\
\hline Tumor size $(\mathrm{cm})$ & 0.013 & $-0.014-0.041$ & 0.339 & & & \\
\hline Moderate/poor differentiation & -0.113 & $-0.322-0.097$ & 0.288 & & & \\
\hline Mixed hepatocellular carcinoma & -0.031 & $-0.295-0.234$ & 0.819 & & & \\
\hline Extrahepatobiliary invasion & 0.134 & $-0.042-0.130$ & 0.135 & & & \\
\hline Resection margin involved & 0.009 & $-0.170-0.189$ & 0.918 & & & \\
\hline$>1$ segment of resection & 0.031 & $-0.234-0.295$ & 0.819 & & & \\
\hline \multicolumn{7}{|l|}{ Biochemistry } \\
\hline $\mathrm{CEA}, \mathrm{ng} / \mathrm{ml}$ & 0.001 & $0.000-0.002$ & 0.050 & & & \\
\hline CA-19-9, x1,000 U/ml & 0.004 & $-0.001-0.008$ & 0.103 & & & \\
\hline Bilirubin, mg/dl & 0.002 & $-0.026-0.030$ & 0.870 & & & \\
\hline $\mathrm{AST}, \mathrm{U} / 1$ & -0.00000257 & $-0.001-0.001$ & 0.997 & & & \\
\hline ALT, U/l & 0.000 & $-0.001-0.001$ & 0.667 & & & \\
\hline
\end{tabular}

CI, confidence interval; HBsAg, hepatitis B virus surface antigen; HCV, hepatitis C virus; ECOG, Eastern Co-operative Oncology Group; CEA, carcinoembryonic antigen; CA-19-9, carbohydrate antigen 19-9; AST, aspartate aminotransferase; ALT, alanine aminotransferase; GALNT14, $\mathrm{N}$-acetylgalactosaminyltransferase 14 .

overall survival using the Cox proportional hazards model. In previous clinical studies, the genotype-prognosis association was revealed to be based on comparison of the 'TT' and 'non-TT' genotypes (including 'TG' and 'GG') (12-14); therefore, the same genotype classification was used in the current study. The median post-resection follow-up time was 14 months (range, 1-180). Age, sex, liver cirrhosis, cholangitis and biliary tree stones did not demonstrate significant associations with overall survival post-resection. By contrast, five tumor characteristics (vessel invasion, perineural invasion, lymph node metastasis, largest tumor size and resection margins), two tumor-associated serum biomarkers (CEA and CA-19-9 levels) and GALNT14 genotypes, revealed significant associations in univariate analysis (Table II). Patients stratified by the 'TT' and 'non-TT' genotypes demonstrated distinguishable survival curves in the Kaplan-Meier plot (log-rank, $\mathrm{P}=0.023$; Fig. 1B). Subsequently, multivariate analysis was performed on these associated factors, excluding CEA and CA-19-9, which had not been assessed in the majority of patients. It was revealed that vascular and perineural invasions are two independent factors associated with overall survival $(\mathrm{P}=0.002$ and $\mathrm{P}=0.001$, respectively), whereas GALNT14 genotypes were not independently associated with overall survival.

Germline GALNT14 genotypes are independently correlated with perineural invasion and lymph node metastasis in resected cholangiocarcinoma. The genotype-prognosis association was exclusively observed in the univariate analysis, and not in the multivariate analysis, which suggested that the genotype may have an unrecognized association with the tumor characteristics. In consideration of this, the present study further investigated the correlations between genotypes and the evaluated clinicopathological parameters, using univariate and multivariate linear regressions. Amongst all the clinicopathological parameters, two tumor characteristics, perineural invasion and lymph node metastasis, were 
determined to be independently associated with the GALNT14 genotype 'TT' (multivariate analysis, $\mathrm{P}=0.035$ and $\mathrm{P}=0.005$, respectively; Table III). The percentage of perineural invasion was significantly higher in patients with the 'TT' genotype, compared with those with a 'non-TT' genotype $(\mathrm{P}=0.004$; Fig. 1C). Similarly, the frequency of lymph node metastasis was significantly higher in patients with the 'TT' genotype, compared with the 'non-TT' genotype ( $\mathrm{P}=0.011$; Fig. 1C).

Subsequently, the genotype distributions ('TT' vs. 'non-TT') were investigated in subgroups of patients stratified by the presence or absence of the two aggressive characteristics, perineural invasion and lymph node metastasis. The distribution of patients with perineural invasion deviated significantly from the reference cohorts, HapMap-CHB and CHD (CHB, $\mathrm{P}=0.049$; CHD, $\mathrm{P}=0.034$ ), where the 'TT' type was particularly enriched (23). No such deviations were identified in patients without perineural invasion $(\mathrm{CHB}, \mathrm{P}=0.144$; $\mathrm{CHD}, \mathrm{P}=0.236$ ). Similarly, the genotype distribution in patients with lymph node metastasis also deviated significantly from the ethnic references (CHB, $\mathrm{P}=0.046$; $\mathrm{CHD}, \mathrm{P}=0.032$ ). No such deviations were identified in patients without lymph node metastasis (CHB, $\mathrm{P}=0.337$; $\mathrm{CHD}, \mathrm{P}=0.501)$.

\section{Discussion}

Aggressive growth of cholangiocarcinoma occurred sporadically with no known major predisposition etiology $(3,4)$. Therefore, it was conjectured in the present study that personal genetic background may contribute to onset, progression and malignant phenotypes. The present study demonstrated that the GALNT14 genotype 'TT' was independently associated with two known predictors of unfavorable prognosis in cholangiocarcinoma: Perineural invasion and lymph node metastasis. The 'TT' genotypes were revealed to be particularly enriched in patients with these aggressive phenotypes, as compared with the ethnic references. Such enrichment may be due to patients with the 'TT' type being more likely to develop these two aggressive tumor characteristics. In the survival analysis, the association between the GALNT14 genotype and overall survival was only observed in univariate analysis, and not in multivariate analysis. It is possible that the tumor characteristics-prognosis association in the multivariate analysis concealed the underlying genotype-prognosis association. As the genotype is determined at birth, while perineural invasion and lymph node metastasis are identified at the time of surgical treatment, a causal association may be inferred that the genotype first affected the development of these two tumor characteristics, which subsequently altered the postoperative prognosis (Fig. 1).

The present study was an extension of previous studies on HCC and cholangiocarcinoma, as a result of their similarities and differences $(3,15,17,18,23,24)$. Cholangiocarcinoma arises from bile duct epithelial cells, whereas HCC originates from hepatocytes $(3,24)$. Cholangiocarcinoma and HCC have fundamental differences in their oncogenic pathways $(3,24)$. HCC is primarily caused by viral hepatitis, including chronic $\mathrm{HBV}$ rather than $\mathrm{HCV}$, dependent on the region of the world (25). By contrast, even in Taiwan, which is a HBV hyperendemic region, the percentage of $\mathrm{HBsAg-positivity} \mathrm{among} \mathrm{patients}$ with cholangiocarcinoma is low $(23.2 \%)$, as compared with in patients with $\mathrm{HCC}(>60 \%)(1,2,26)$. An additional difference is that $\mathrm{HCC}$ often develops from a cirrhotic background; however, the majority of patients with cholangiocarcinoma in the present study were non-cirrhotic (83\%) (26). Despite these differences, certain HCC and cholangiocarcinoma cases have overlapping histology patterns, demonstrating mixed tissue types (3). As GALNT14 encodes an enzyme that catalyzes the O-glycosylation of numerous proteins, it is possible that differential O-glycosylation environments associated with various GALNT14 genotypes may result in the distinct tumor characteristics of cholangiocarcinoma and HCC (19). Further studies focusing on the underlying molecular mechanisms are required to clarify this point.

In conclusion, patients with the GALNT14 genotype 'TT' are associated with two aggressive tumor characteristics: Perineural invasion and lymph node metastasis. This genotype was therefore associated with an unfavorable overall survival.

\section{Acknowledgements}

The present study was supported by the Chang Gung Memorial Hospital (grant nos. CMRPG1B0571, CIRPG3B0032, CLRPG3C0011 and CLRPG3C0012). The authors thank Miss Hui-Chin Chen, Dr Ji-Wei Lin, Miss Yen-Ling Chuang, Miss Wen-Hsin Kuo, Miss Hsiao-Chih Yu, Miss Hsiu-Ting Wang and Miss May-Ling Tsao from the Liver Research Center, Chang Gung Memorial Hospital for their excellent technical assistance.

\section{References}

1. Khan SA, Davidson BR, Goldin RD, Heaton N, Karani J, Pereira SP, Rosenberg WM, Tait P, Taylor-Robinson SD, Thillainayagam AV, et al: Guidelines for the diagnosis and treatment of cholangiocarcinoma: An update. Gut 61: 1657-1669, 2012.

2. Bridgewater J, Galle PR, Khan SA, Llovet JM, Park JW, Patel T, Pawlik TM and Gores GJ: Guidelines for the diagnosis and management of intrahepatic cholangiocarcinoma. J Hepatol 60: 1268-1289, 2014.

3. Blechacz B and Gores GJ: Cholangiocarcinoma: Advances in pathogenesis, diagnosis, and treatment. Hepatology 48: 308-321, 2008.

4. Bragazzi MC, Carpino G; Venere R, Semeraro R, Gentile R, Gaudio E and Alvaro D: Cholangiocarcinoma: Epidemiology and risk factors. Transl Gastrointest Cancer 1: 21-32, 2012.

5. Shaib YH, Davila JA, Henderson L, McGlynn KA and El-Serag HB: Endoscopic and surgical therapy for intrahepatic cholangiocarcinoma in the United States: A population-based study. J Clin Gastroenterol 41: 911-917, 2007.

6. Cheng CT, Chu YY, Yeh CN, Huang SC, Chen MH, Wang SY, Tsai CY, Chiang KC, Chen YY, Ma MC, et al: Peritumoral SPARC expression and patient outcome with resectable intrahepatic cholangiocarcinoma. Onco Targets Ther 8: 1899-1907, 2015.

7. Shirai K, Ebata T, Oda K, Nishio H, Nagasaka T, Nimura Y and Nagino M: Perineural invasion is a prognostic factor in intrahepatic cholangiocarcinoma. World J Surg 32: 2395-2402, 2008.

8. Chen TC, Jan YY and Yeh TS: K-ras mutation is strongly associated with perineural invasion and represents an independent prognostic factor of intrahepatic cholangiocarcinoma after hepatectomy. Ann Surg Oncol 19 (Suppl 3): S675-S681, 2012.

9. Nathan H, Aloia TA, Vauthey JN, Abdalla EK, Zhu AX, Schulick RD, Choti MA and Pawlik TM: A Proposed staging system for intrahepatic cholangiocarcinoma. Ann Surg Oncol 16: 14-22, 2009.

10. Okabayashi T, Yamamoto J, Kosuge T, Shimada K, Yamasaki S, Takayama $\mathrm{T}$ and Makuuchi M: A new staging system for mass-forming intrahepatic cholangiocarcinoma: Analysis of preoperative and postoperative variables. Cancer 92: 2374-2383, 2001. 
11. Chaiteerakij R, Harmsen WS, Marrero CR, Aboelsoud MM Ndzengue A, Kaiya J, Therneau TM, Sanchez W, Gores GJ and Roberts LR: A new clinically based staging system for perihilar cholangiocarcinoma. Am J Gastroenterol 109: 1881-1890, 2014.

12. Paul A, Kaiser GM, Molmenti EP, Schroeder T, Vernadakis S, Oezcelik A, Baba HA, Cicinnati VR and Sotiropoulos GC: Klatskin tumors and the accuracy of the Bismuth-Corlette classification. Am Surg 77: 1695-1699, 2011.

13. Matsuo K, Rocha FG, Ito K, D'Angelica MI, Allen PJ, Fong Y, Dematteo RP, Gonen M, Endo I and Jarnagin WR: The Blumgart preoperative staging system for hilar cholangiocarcinoma: Analysis of resectability and outcomes in 380 patients. J Am Coll Surg 215: 343-355, 2012.

14. Zervos EE, Osborne D, Goldin SB, Villadolid DV, Thometz DP, Durkin A, Carey LC and Rosemurgy AS: Stage does not predict survival after resection of hilar cholangiocarcinomas promoting an aggressive operative approach. Am J Surg 190: 810-815, 2005.

15. Liang KH, Lin CC and Yeh CT: GALNT14 SNP as a potential predictor of response to combination chemotherapy using 5-FU, mitoxantrone and cisplatin in advanced HCC Pharmacogenomics 12: 1061-1073, 2011.

16. Lin WR, Hsu CW, Chen YC, Chang ML, Liang KH, Huang YH and Yeh CT: GALNT14 genotype, $\alpha$-fetoprotein and therapeutic side effects predict post-chemotherapy survival in patients with advanced hepatocellular carcinoma. Mol Clin Oncol 2: 630-640, 2014.

17. Yeh CT, Liang KH, Lin CC, Chang ML, Hsu CL and Hung CF: A single nucleotide polymorphism on the GALNT14 gene as an effective predictor of response to chemotherapy in advanced hepatocellular carcinoma. Int J Cancer 134: 1214-1224, 2013.

18. Liang KH, Lin CL, Chen SF, Chiu CW, Yang PC, Chang ML, Lin CC, Sung KF, Yeh C, Hung CF, et al: GALNT14 genotype effectively predicts the therapeutic response in unresectable hepatocellular carcinoma treated with transcatheter arterial chemoembolization. Pharmacogenomics 17: 353-366, 2016.
19. Wagner KW, Punnoose EA, Januario T, Lawrence DA, Pitti RM, Lancaster K, Lee D, von Goetz M, Yee SF, Totpal K, et al: Death-receptor O-glycosylation controls tumor-cell sensitivity to the proapoptotic ligand Apo2L/TRAIL. Nat Med 13: 1070-1077, 2007.

20. De Mariano M, Gallesio R, Chierici M, Furlanello C, Conte M, Garaventa A, Croce M, Ferrini S, Tonini GP and Longo L: Identification of GALNT14 as a novel neuroblastoma predisposition gene. Oncotarget 6: 26335-26346, 2015.

21. Shamseldin HE, Tulbah M, Kurdi W, Nemer M, Alsahan N, Al Mardawi E, Khalifa O, Hashem A, Kurdi A, Babay Z, et al: Identification of embryonic lethal genes in humans by autozygosity mapping and exome sequencing in consanguineous families. Genome Biol 16: 116, 2015.

22. Ihle MA, Fassunke J, König K, Grünewald I, Schlaak M, Kreuzberg N, Tietze L, Schildhaus HU, Büttner R and Merkelbach-Bruse S: Comparison of high resolution melting analysis, pyrosequencing, next generation sequencing and immunohistochemistry to conventional Sanger sequencing for the detection of p.V600E and non-p.V600E BRAF mutations. BMC Cancer 14: 13, 2014

23. Liang KH, Yang PC and Yeh CT: Genotyping the GALNT14 gene by joint analysis of two linked single nucleotide polymorphisms using liver tissues for clinical and geographical comparisons. Oncol Lett 8: 2215-2220, 2014.

24. Inokawa Y, Inaoka K, Sonohara F, Hayashi M, Kanda M and Nomoto S: Molecular alterations in the carcinogenesis and progression of hepatocellular carcinoma: Tumor factors and background liver factors. Oncol Lett 12: 3662-3668, 2016.

25. Sherman M: Hepatocellular carcinoma: Epidemiology, surveillance, and diagnosis. Semin Liver Dis 30: 3-16, 2010.

26. Yeh CT, Huang YH, Liang KH, Chang ML, Hsu CW, Chen YC, Chen TC, Yeh TS and Lee WC: Segregation of signaling proteins as prognostic predictors for local recurrence and distant metastasis in hepatocellular carcinoma. Int J Oncol 44: 491-504, 2014. 